\title{
Development of a near-infrared high-resolution spectrograph (WINERED) for a survey of bulge stars
}

\author{
T. Tsujimoto ${ }^{1}$, N. Kobayashi ${ }^{2}$, C. Yasui ${ }^{2}$, S. Kondo ${ }^{2}$, A. Minami $^{2}$, \\ K. Motohara ${ }^{2}$, Y. Ikeda ${ }^{3}$ and N. Gouda ${ }^{1}$ \\ ${ }^{1}$ National Astronomical Observatory, Mitaka, Tokyo 181-8588, Japan \\ email: taku.tsujimoto@nao.ac.jp \\ ${ }^{2}$ Institute of Astronomy, University of Tokyo, Mitaka, Tokyo 181-0015, Japan \\ ${ }^{3}$ Photocoding, Sagamihara, Kanagawa 229-1104, Japan
}

\begin{abstract}
We are developing a new near-infrared high-resolution $(\mathrm{R}[\max ]=100,000)$ and high-sensitive spectrograph WINERED, which is specifically customized for short NIR bands at 0.9-1.35 $\mu \mathrm{m}$. WINERED employs an innovative optical system; a portable design and a warm optics without any cold stops. The planned astrometric space mission JASMINE will provide precise positions, distances, and proper motions of the bulge stars. The missing components, the radial velocity and chemical composition will be measured by WINERED. These combined data brought by JASMINE and WINERED will certainly reveal the nature of the Galactic bulge. We plan to complete this instrument for observations of single objects by the end of 2008 and to attach it to various $4-10 \mathrm{~m}$ telescopes as a PI-type instrument. We hope to upgrade WINERED with a multi-object feed in the future for efficient survey of the JASMINE bulge stars.
\end{abstract}

Keywords. Galaxy: bulge, infrared: stars, stars: abundances, stars: atmospheres, instrumentation: spectrographs, techniques: spectroscopic, techniques: radial velocities

We are developing a new NIR high-resolution spectrograph WINERED (=Warm INfrared Echelle spectrograph to Realize Extreme Dispersion) by employing two novel approaches (Ikeda et al. 2006). First, WINERED employs warm optics with no cold stop, which can be realized by limiting the wavelength range to short NIR bands of 0.9 $1.35 \mu \mathrm{m}$. Second, WINERED employs an immersion grating of $\mathrm{ZnSe}$ or $\mathrm{ZnS}$, resulting in a high resolving power of $R_{\max }=100,000$, despite its compactness $(1,500 \times 500 \times 500 \mathrm{~mm})$. These two approaches make WINERED portable and easy to build, align, and maintain. Therefore, the total cost and time can be significantly reduced as compared with an entirely cooled classical echelle spectrograph.

\section{Warm optics}

In the short NIR region $(\lambda \leqslant 1.35 \mu \mathrm{m})$, the ambient thermal and sky backgrounds are negligible if compared to the readout noise (Ikeda et al. 2006, Kondo et al. 2006). It means that the cooling of entire optics is not necessary as long as we remain in this short NIR region. WINERED employs warm (room temperature) optics except for the camera system that includes the detector array (Fig. 1). The narrow wavelength range significantly increases the performance of the AR coating on the optical elements, e.g., $R<1 \%$ per surface is possible, while $R>5 \%$ per surface is inevitable at some wavelengths for $1.0-5.5 \mu \mathrm{m}$ broad band AR. This leads to a significant throughput improvement for WINERED which uses a large number of refractive optics for the aberration-free design. 


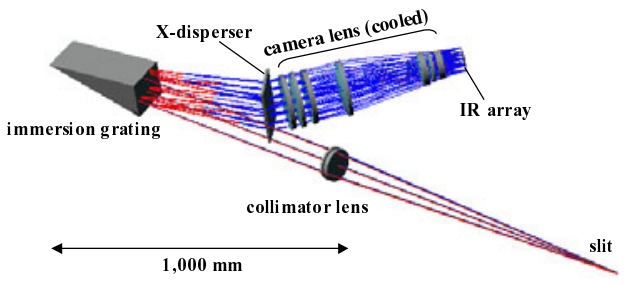

Figure 1. Optical layout of WINERED

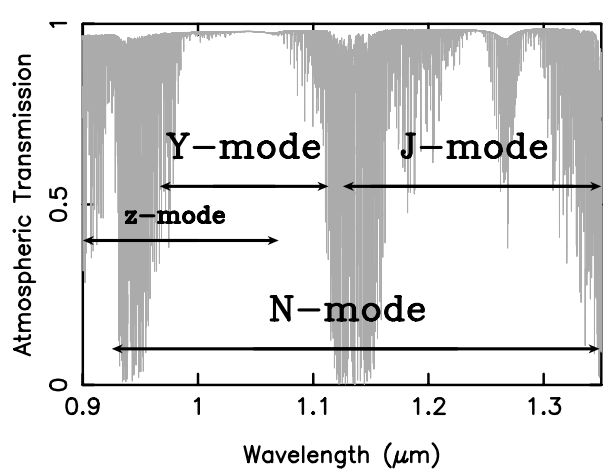

Figure 2. Wavelength coverage of WINERED

\section{Immersion grating}

An immersion grating of an infrared material with a high refractive index $(n>2)$ can provide a higher resolution and reduce the size of the collimated beam to $1 / n$ for the same resolving power. The main disperser, WINERED uses an immersion grating made of ZnSe $(n \sim 2.4)$ or multi-spectral grade $\mathrm{ZnS}(n \sim 2.3)$, which has a high refractive index and little absorption at NIR wavelengths. By adopting the immersion grating, WINERED can achieve a high resolving power within a very small instrumental volume and compact optics. Our current trial of groove processing on ZnSe blocks uses a nano precision flycutting technique at the Lawrence Livermore National Laboratory (Kuzmenko 2006), which was proved to be quite successful.

\section{Optical system}

WINERED is a cross-dispersed-type echelle spectrograph that has a refractive collimator, a ZnSe immersion grating, two (blue and red) Volume Phase Holographic gratings (VPHs) for the cross-disperser, and a cryogenic refractive lens system for the camera optics (Fig. 1). We employed this simplest classical optical configuration because it provides the best balance between the system throughput, alignment tolerances, and image quality (high spectral resolution). See Yasui et al. (2006) for details of the optical design.

Because we anticipate a long lead time for the development of the $\mathrm{ZnSe} / \mathrm{ZnS}$ immersion grating, we plan to install a classical reflection echelle grating with a groove density of 31.6 lines $\mathrm{mm}^{-1}$ and a blaze angle of $63.9 \mathrm{deg}$, which will be made switchable to the immersion grating. This normal echelle mode can have a spectrum between $0.9-1.35 \mu \mathrm{m}$ simultaneously with the resolving power of $R_{\max }=28,300$ in one exposure (N-mode in Fig. 2). This mode serves as a full-science mode as well as a practice mode prior to the installation of the immersion grating. We plan to use mostly this mode to cover stellar metal absorption lines in this wavelength range for the JASMINE pre-study of bulge stars.

\section{References}

Ikeda, Y., et al. 2006, SPIE, 6269, 62693T

Kondo, S., et al. 2006, SPIE, 6269, 62694O

Kuzmenko, P. 2006, SPIE, 6273, 62733S

Yasui, C., et al. 2006, SPIE, 6269, 62694P 